High confidence optical diagnosis agreed with histopathology in $78.2 \%(366 / 468)$ of cases and disagreed in $21.8 \%$ $(102 / 468)$. In cases of disagreement, the initial histopathology was reviewed and $7.8 \%(8 / 102)$ were due to histopathology error of which $3.9 \%(4 / 102)$ corrected on second review and $3.9 \%(4 / 102)$ corrected with deeper levels.

There were no polyp cancers and 1 case of high grade dysplasia.

Conclusions Although the majority of errors in optical diagnosis were related to incorrect high confidence calls a significant number were due to histopathology error. Change in practice to routinely perform additional deeper levels (ie 6 levels instead of 3) for small polyps appears to reduce this error rate by $\sim 50 \%$. Optical diagnosis errors may be reduced by increasing the threshold for assignment of high confidence.

\section{HTU-4 COMPARISON OF POST COLONOSCOPY CANCER RATES IN SCREENING \& SYMPTOMATIC SERVICES IN NHS GREATER GLASGOW}

Jennifer Tham*, Eliana Saffouri, Jack Winter. Glasgow Royal Infirmary, Glasgow, UK

\subsection{6/gutjnl-2021-BSG.51}

Introduction The Post-Colonoscopy Cancer Rate at 3 years (PCCR-3yr) is a key indicator of quality of a service. The bowel screening programme (BCSP) in NHS England has reported PCCR-3 of $3.6 \%{ }^{1}$ The bowel screening programme in Scotland has key differences to the English BCSP and does not rely on a specific accreditation programme with examination for screeners, although has concentrated investment in access by offering screening to age 50-74 years at outset. Our aim was to ascertain the PCCR in the bowel screening service in NHS Greater Glasgow and Clyde (the largest Scottish Health Board - population 1.14 million) during the period 2011-15, and compare with the rate in the symptomatic service for a similar age range (50-77yrs).

Method For each year within the study period, the total number of known cancer diagnoses was ascertained from cancer audit data submissions, identifying the 'true positive' colonoscopies. Cancer audit data was then linked to identify cases where a cancer was detected between 6 and 36 months after the index colonoscopy, giving the number of 'false negative' colonoscopies. Post colonoscopy cancer rate was then determined by expressing the number of 'false negative' colonoscopies as a percentage of the sum of 'true positive' and 'false negative' colonoscopies. The rates of post colonoscopy cancers between the screening and non-screening pathways were compared using the chi squared test.

Results There were 1909 true positive colonoscopies in the investigation period for the entire population. We found 102 cases of PCCR-3y, giving a rate of 5.2\% (95\% CI 4.6-5.6\%). $678 / 2011(34 \%)$ of all bowel cancers were screen detected. PCCR-3yr for the screening service was 4.4\% (95\% CI 3.6$5.2 \%)$, which was lower than 5.5\% (95\% CI 4.8-6.1\%) for the symptomatic service but not statistically different.

Conclusion The overall PCCR-3yr for NHS GGC between 2011 and 2015 of $5.2 \%$ is similar to rates reported for England between 2015 and 2013. Post colonoscopy cancer rates for screening colonoscopy in NHS GGC were slightly lower than for the symptomatic service but not statistically different. Our rates were higher than rates reported for the English BCSP, but within the threshold of $5.5 \%{ }^{1}$ that has been proposed by some investigators. This is the first report of PCCR-3yr in NHS Scotland and we believe that regular continuous audit of this important quality indicator should be replicated all Scottish boards. In our service, PCCR-3yr rates appear acceptable within NHS GGC and are slightly better for bowel screening compared with non-screening colonoscopy.

\section{REFERENCE}

1. Burr et al BMJ 2019

\section{HTU-5 NATIONAL SERVICE EVALUATION OF THE TWO WEEK WAIT UPPER GI CANCER REFERRAL PATHWAY}

Umair Kamran*, Dominic King, Matthew Banks, Sophie Barker, Matthew Caffrey, Danny Cheung, James Evans, Mark Fox, Michael Glynn, John Greenaway, Sanjay Gupta, Srisha Hebbar, Miriam Jones, Sudarshan Kadri, David Mitchell, David Nylander, Rupert Ransford, Sharan Shetty, Tony Tham, Matthew Williams, Nigel Trudgill. Upper GI cancer two week wait study group, UK

\subsection{6/gutjnl-2021-BSG.52}

Introduction British Society of Gastroenterology (BSG) guidance on endoscopy during recovery from the COVID pandemic (April 2020) recommended that two week wait (2WW) referrals are triaged, with patients risk stratified for endoscopy or other investigation. We have prospectively evaluated the 2WW upper gastrointestinal (UGI) cancer pathway and its outcomes following this guidance.

Methods Data were collected at telephone triage by consultants and nurse endoscopists between May 2020 and February 2021 in 19 centres across the UK and recorded on a standardised data collection tool, which included recommendations on the timing of endoscopy based on the BSG recovery document. This project was supported by the BSG Clinical Services and Standards Committee.

Results Data for 1793 UGI 2WW referrals were received: median age 63 (IQR 51-74), 58\% female. Dysphagia and odynophagia were the commonest reasons (83\%) for referral. Other symptoms included dyspepsia (55\%), weight loss (32\%), globus (3\%), and anaemia (3\%). $15.8 \%$ of $2 \mathrm{WW}$ referrals were downgraded at triage to routine endoscopy $(6.6 \%)$ or no investigation at all (9.2\%). 56\% were triaged to $2 \mathrm{WW}$ endoscopy; $19.6 \%$ to urgent (non-2WW) endoscopy; $4.7 \%$ to urgent CT scan; and $3.8 \%$ to barium swallow.

$6.3 \%$ had UGI cancer $(5.2 \%$ oesophageal, $1.1 \%$ gastric) and $0.9 \%$ had cancer at other sites (6 colorectal, 2 lung, 2 breast, 2 hypopharyngeal, 1 pancreatic and 2 unknown primary). Endoscopy results were available for 1387 patients (97.5\% of all endoscopy pathways). The prevalence of UGI

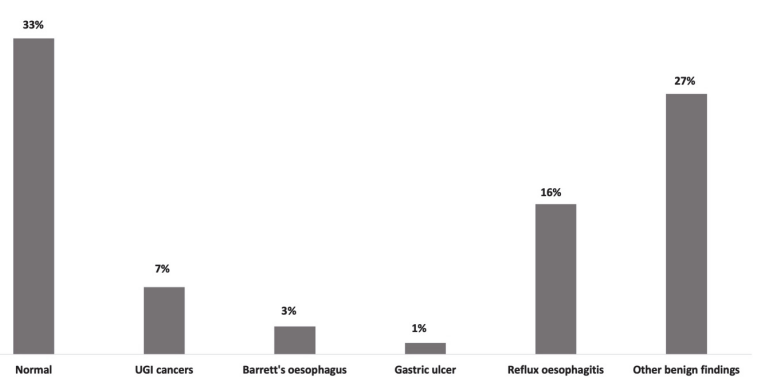

Abstract HTU-5 Figure 1 The median interval from triage to endoscopy was: 12 days (IQR 8-18) for 2WW; 14 days (10-26) for urgent (non-2WW); and 17 days (9-38) for routine endoscopy 
cancer in different triage categories was: 2WW 9\%; urgent (non-2WW) endoscopy 3\%; urgent CT 8.5\%; and routine endoscopy $1 \%$. Triage based on the BSG recovery guidance was $97 \%$ sensitive and 19\% specific for upper GI cancer (at 2WW or urgent endoscopy or CT scan), with a negative predictive value of $99 \%$ and a positive predictive value of $8 \%$. A summary of all endoscopy findings from the service evaluation is presented in figure 1.

Conclusions Triage based on the BSG recovery guidance was $97 \%$ sensitive with a negative predictive value of $99 \%$ in diagnosing UGI cancer at $2 \mathrm{WW}$ or urgent endoscopy or CT scan. $6.6 \%$ of $2 \mathrm{WW}$ referrals were safely investigated routinely and over $9 \%$ of $2 \mathrm{WW}$ referrals required no investigation at all following triage. These findings should guide reform of the upper GI 2WW pathway to reduce the burden on endoscopy during and after the COVID pandemic.

\section{IBD}

\section{HMO-1 VARIATION IN IBD CARE AND EDUCATION ACROSS EUROPE RESULTS FROM A PAN-EUROPEAN SURVEY}

Viper collaborative Viper Collaborative Aileen Eek, Adonis A Protopapas, Anthea Pisani, Brigida Barberio, Catarina Frias-Gomes, Daniele Noviello, Dmytro Oliinyk, Eduard Brunet, Florian Tran, Gabriele Dragoni, Georgiana-Emmanuela Gîlcă-Blanariu, Gorm Roager Madsen, Haluk Tarik Kani, Hubert Zatorski, lago Rodríguez-Lago, Ivana Mikolasevic, Lauranne AAP Derikx, Leah Gilroy, Maria Miasnikova, Marie Truyens, Matthias Lessing, Marcin Włodarczyk, Philip R Harvey, Nicolas Benech, Tiago Lima Capela, Tom Konikoff, Vaidota Maksimaityte, Vita Skuja, Vladimir Milivojevic, ${ }^{1} J a n$ Kral, ${ }^{2}$ Radislav Nakov, ${ }^{3}$ Bram Verstockt, ${ }^{4}$ Jonathan Segal. ${ }^{1}$ Institute for Clinical and Experimental Medicine, Prague, Czech Rep; ${ }^{2}$ Tsaritsa Yoanna University Hospital, Sofia, Bulgaria; ${ }^{3}$ University Hospitals Leuven, Leuven, Belgium; ${ }^{4}$ Hillingdon Hospital, Uxbridge, UK

\subsection{6/gutjnl-2021-BSG.53}

Background 2.5 million people in Europe are diagnosed with IBD. IBD affects quality of life, but also has important consequences for health systems. It remains unknown if there are variations in IBD care across Europe and to help address this question, we conducted this European Variation In ibd PracticE suRvey (VIPER) to study potential differences.

Methods This trainee-initiated survey, run through SurveyMonkey ${ }^{\circledR}$, consisted of 47 questions inquiring basic demographics, IBD training and clinical care. The survey was distributed through social media and national GI societies from December 2020 - January 2021. Results were compared according to GDP per capita, for which countries were divided into 2 groups (low/high income, according to the World Bank).

Results There were 1268 participants from 39 European countries. Most of the participants are specialists (65.3\%), followed by fellows in training $(>/<3$ years, 19.1\%, 15.6\%). Majority of the responders are working in academic institutions $(50.4 \%)$, others in public/district hospitals $(33.3 \%)$ or private practices $(16.3 \%)$.

Despite significant differences in access to IBD-specific training between high (56.4\%) and low (38.5\%) GDP countries $(\mathrm{p}<0.001)$ the majority of clinicians felt comfortable in treating IBD $(77.2 \%$ vs $72.0 \%, \mathrm{p}=0.04)$. Interestingly, a difference in availability of dedicated IBD units could be observed $(58.5 \%$ vs $39.7 \%, \mathrm{p}<0.001)$, as well as an inequality in multidisciplinary meetings $(72.6 \%$ vs $40.2 \%, \mathrm{p}<0.001)$, which often take place on a weekly basis (53.0\%). In high GDP countries, IBD nurses are more common (86.2\%) than in low GDP countries $(36.0 \%, \mathrm{p}<0.001)$, which is mirrored by differences in nurse-led IBD clinics $(40.6 \%$ vs $13.8 \%, \mathrm{p}<0.001)$. IBD

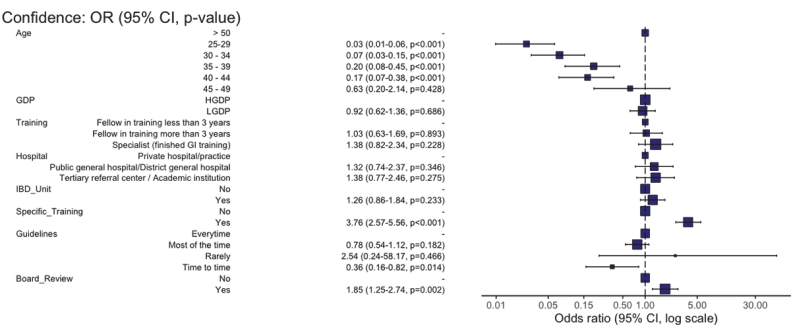

Abstract HMO-1 Figure 1 Factors associated with confidence on multivariate analysis

dieticians $(32.4 \%$ vs $16.6 \%)$ and psychologists $(16.7 \%$ vs $7.5 \%)$ are mainly present in high GDP countries $(\mathrm{p}<0.001)$.

On multivariate analysis (Abstract HMO1 Figure 1) GDP was not a factor that dictated confidence in treating patients with IBD. Those that had experienced specific IBD training were more than 3 times more likely to report confidence in treating IBD patients. Furthermore, there was a direct correlation with confidence related to the quantity of patients seen per week with each additional 10 patients improving confidence by a factor of 10 . These factors are important as they are easily modifiable targets that can help improve confidence across Europe in managing patients with IBD.

Treat-to-target approaches are implemented everywhere (85.0\%), though access to biologicals and small molecules differs significantly. Almost all (94.7\%) use faecal calprotectin for routine monitoring, whereas half also use intestinal ultrasound (47.9\%).

Conclusion A lot of variability in IBD practice exists across Europe, with marked differences between high vs low GDP countries. Further work is required to help address some of these inequalities, aiming to improve and standardise IBD care across Europe.

\section{HMO-2 ADHERENCE AND DISCONTINUATION OF ORAL 5- AMINOSALICYLIC ACID AMONGST ADOLESCENTS AND YOUNG ADULTS WITH ULCERATIVE COLITIS}

\footnotetext{
1,2Nishani Jayasooriya*, 1,2Richard Pollok, 1,2 Jonathan Blackwell, ${ }^{3}$ Irene Petersen,
} ${ }^{2}$ Alex Bottle, ${ }^{2}$ Hanna Creese, ${ }^{2}$ Sonia Saxena. 'St George's University Hospital, London, London, UK; ${ }^{2}$ Imperial College London, Department of Primary Care and Public Health, London, UK; ${ }^{3}$ University College London, Institute of Epidemiology and Health, London, UK

\subsection{6/gutjnl-2021-BSG.54}

Background Adherence to maintenance 5-amino-salicylic-acid (5-ASA) therapy is associated with better health and quality of life of adolescents and young adults (AYA) diagnosed with Ulcerative Colitis (UC). However, little is known about rates of adherence and how often AYA discontinue oral 5-ASA treatment.

Aims To determine rates and predictors of oral 5-ASA adherence and risk of discontinuation amongst AYA diagnosed with UC.

Methods A retrospective data analysis was performed within the UK Clinical Practice Research Datalink amongst AYA diagnosed with UC between 1998 and 2016 and and starting on oral 5-ASA treatment between the ages of 10 to 24 years. The proportion of individuals discontinuing treatment (first prescription gap of $\geq 90$ days) in the first year of treatment and the median time until a first 90-day gap was estimated using Kaplan-Meier analysis. Adherence, measured as 